\title{
Análise da variação espacial e sazonal e de tendências na precipitação da região sul do Brasil
}

\section{Analysis of space and seasonal variation and trends in the precipitation of the southern region of Brazil}

\author{
Fabiane N. Gonçalves ${ }^{1, *}$ e Álvaro J. Back ${ }^{1,2}$ \\ ${ }_{1}$ Programa de Pós-Graduação em Ciências Ambientais - Universidade do Extremo Sul Catarinense (UNESC), Criciúma, SC, Brasil \\ 2 Empresa de Pesquisa Agropecuária e Extensão Rural de Santa Catarina, Epagri, Urussanga, SC, Brasil \\ (*E-mail: fabianenunes@yahoo.com.br) \\ http://dx.doi.org/10.19084/RCA17204
}

Recebido/received: 2017.08.15

Recebido em versão revista/received in revised form: 2017.10.25

Aceite/accepted: 2018.02.20

\section{R E S U M O}

A distribuição da precipitação tem grande influência na produção agrícola e na economia da região Sul do Brasil. Este estudo teve como objetivo avaliar a distribuição espacial e sazonal e as tendências nas séries de precipitação da região Sul do Brasil. Para o efeito, foram utilizados dados de 197 estações pluviométricas localizadas na Região Sul do Brasil, que apresentaram mais de 32 anos de dados nas séries anuais no período de 1976 a 2015. Foram determinadas as séries anual e sazonal de precipitação e do Índice de Concentração da Precipitação (ICP). A tendência nas séries consideradas foi avaliada pelo teste de Mann-Kendall ao nível de significância de 5\%. Foram apresentados os mapas evidenciando a variação espacial e sazonal tanto da precipitação quanto do ICP. Os resultados demonstram que as séries podem ser consideradas estacionárias, visto que mais de $83 \%$ das estações pluviométricas não apresentaram tendências significativas de aumento ou de redução no volume de precipitação, nem na concentração de chuva sobre a região em estudo. Em termos de sazonalidade da precipitação, também não ocorreram aí tendências significativas, embora fossem detetadas algumas variações sazonais e espaciais, de modo que no verão há maior número de estações que apresentam tendências de aumento da precipitação, sendo que no outono a tendência é de redução.

Palavras-chave: séries climáticas; chuvas; Mann-Kendall; variabilidade sazonal e espacial

\begin{abstract}
A B S T R A C T
The precipitation distribution has a great influence on the agricultural production and economy of the southern region of Brazil. This study aimed to evaluate the spatial and seasonal distribution and trends in the precipitation series of the southern region of Brazil. For this purpose, we used data from 197 rain gauges located in the Southern Region of Brazil, which presented more than 32 years of data in the annual series from 1976 to 2015. The annual and seasonal precipitation series and the Precipitation Concentration Index (PCI) were obtained. The trend in the series was evaluated by the Mann-Kendall test at a significance level of 5\%. The maps showing the spatial and seasonal variation of both precipitation and PCI were presented. The results show that the series can be considered stationary, since more than $83 \%$ of the rainfall stations did not present significant trends of increase or reduction in the volume of precipitation nor in the concentration of rain on the region under study. In terms of seasonality of precipitation, there were also no significant trends there, although some seasonal and spatial variations were detected, so that in summer there is a greater number of seasons that show increasing precipitation trends, and in autumn the tendency is for reduction.
\end{abstract}

Keywords: climatic series; rainfall; Mann-Kendal; seasonal and spatial variability. 


\section{INTRODUÇÃO}

O clima de uma região tem influência sobre os ecossistemas, na distribuição da vegetação, na formação das paisagens e, na atividade económica. Dentre as variáveis climáticas, a precipitação, assim como a temperatura, são os elementos que atuam de maneira mais direta na configuração da natureza. A variabilidade da distribuição espacial e temporal da precipitação condiciona os ciclos agrícolas e outras atividades humanas. Essa variabilidade das precipitações está relacionada com a dinâmica geral da atmosfera e do relevo da região. Monteiro (2001) afirma que o relevo de Santa Catarina contribui fundamentalmente na distribuição diferenciada da precipitação em distintas áreas do Estado. A influência do relevo na distribuição das chuvas na região Sul do Brasil também foi destacada por Cera et al. (2009), Marengo et al. (2009) e Grimm (2009).

A vulnerabilidade da região sul do Brasil aos impactos que as mudanças climáticas geram sobre a agricultura trazem preocupações, sejam elas sobre sua subsistência ou sobre sua base económica (Pellegrino et al., 2007). Além disso, segundo Ely et al. (2003), os efeitos adversos da precipitação em ambientes rurais implicam na perda parcial ou total de safras, comprometendo assim o mercado, o desemprego e a segurança alimentar.

O sul do Brasil possui boa parte da sua economia associada ao agronegócio, e variabilidade natural climática que atinge a região, pode determinar anos de prejuízo e anos favoráveis à produção agrícola (Cera \& Ferraz, 2015). No Rio Grande do Sul, o clima é um dos fatores responsáveis por quebras de safras e, consequentemente, redução de lucros. Em Santa Catarina destacam-se os danos decorrentes dos períodos de estiagem e seca, causando prejuízos principalmente aos setores agrícolas e pecuários (UFSC, 2016)

A necessidade de compreender as mudanças climáticas e seus efeitos sobre o ambiente natural reforça a importância dos estudos de tendências em séries temporais, dada a incerteza presente na dinâmica dos processos hidrológicos.

Testes estatísticos para análise de tendências em séries de observações hidrológicas são encontrados na literatura e divididos em testes paramétricos e não paramétricos. Os testes paramétricos não são eficientes para muitas séries hidrológicas, visto que necessitam assumir a independência dos dados e muitas vezes uma distribuição normal. Muitas séries hidrológicas raramente seguem uma distribuição normal. Nos métodos não paramétricos, menos suposições sobre a distribuição da variável em estudo precisam ser feitas. Com esses métodos, não é necessário assumir uma distribuição de probabilidades (Queiroz, 2013).

Back (2001) descreveu e aplicou diferentes testes estatísticos para identificação de tendências em séries climáticas de Urussanga, Santa Catarina. O autor utilizou os testes Run, de Mann-Kendall e de Pettitt e a análise de regressão.

Pinheiro et al. (2013), ao analisarem a tendência das séries temporais de precipitação de 18 estações pluviométricas da região sul do Brasil, com amostras superiores a 40 anos, encontraram tendências estatisticamente significativas ao nível de 5\% de aumento dos totais precipitados em 16 estações. Haylock et al. (2006), também encontraram tendências de aumento na intensidade e frequência de dias com chuvas intensas no sul do Brasil. Já Silva et al. (2010), encontraram tendências positivas e negativas no volume de precipitações, sendo que há destaque para tendências positivas significativas desde o centro do Rio Grande do Sul até o Paraná.

A avaliação da distribuição da precipitação pode ser realizada com a utilização de índices climáticos, que podem ser usados na classificação e interpretação dos resultados. O Índice de Concentração da Precipitação ICP (ou PCI - Precipitation Concentration Index) foi proposto por Oliver (1980) com intuito de definir a variabilidade temporal das chuvas durante o ano.

De Luis et al. (2011) afirmam que esse índice permite compreender o comportamento da concentração da chuva num espaço e uma vez que a chuva influencia diretamente na recarga e no fluxo das águas subterrâneas, o conhecimento espacial e concentração da mesma se torna indispensável.

Deste modo, o objetivo deste trabalho é analisar a distribuição espacial e sazonal bem como a presença de tendências nas séries de precipitação e de ICP da região Sul do Brasil. 


\section{MATERIAL E MÉTODOS}

A área de estudo deste trabalho é a região Sul do Brasil, onde foram selecionadas 197 estações pluviométricas com dados diários da Rede Hidrológica da Agência Nacional de Águas (ANA, 2009), sendo que 69 estações se localizam no estado do Paraná, 75 em Santa Catarina e 53 no estado do Rio Grande do Sul (Figura 1). Na seleção das estações adotou-se o critério de utilizar as estações com mais de 32 anos de dados nas séries anuais dentro do período de 1976 a 2015.

As falhas nas séries mensais foram preenchidas pelo método de ponderação regional (Tucci, 2004), onde foram selecionadas três estações pluviométricas no entorno da estação com falha, com características climáticas próximas àquelas a serem preenchidas.

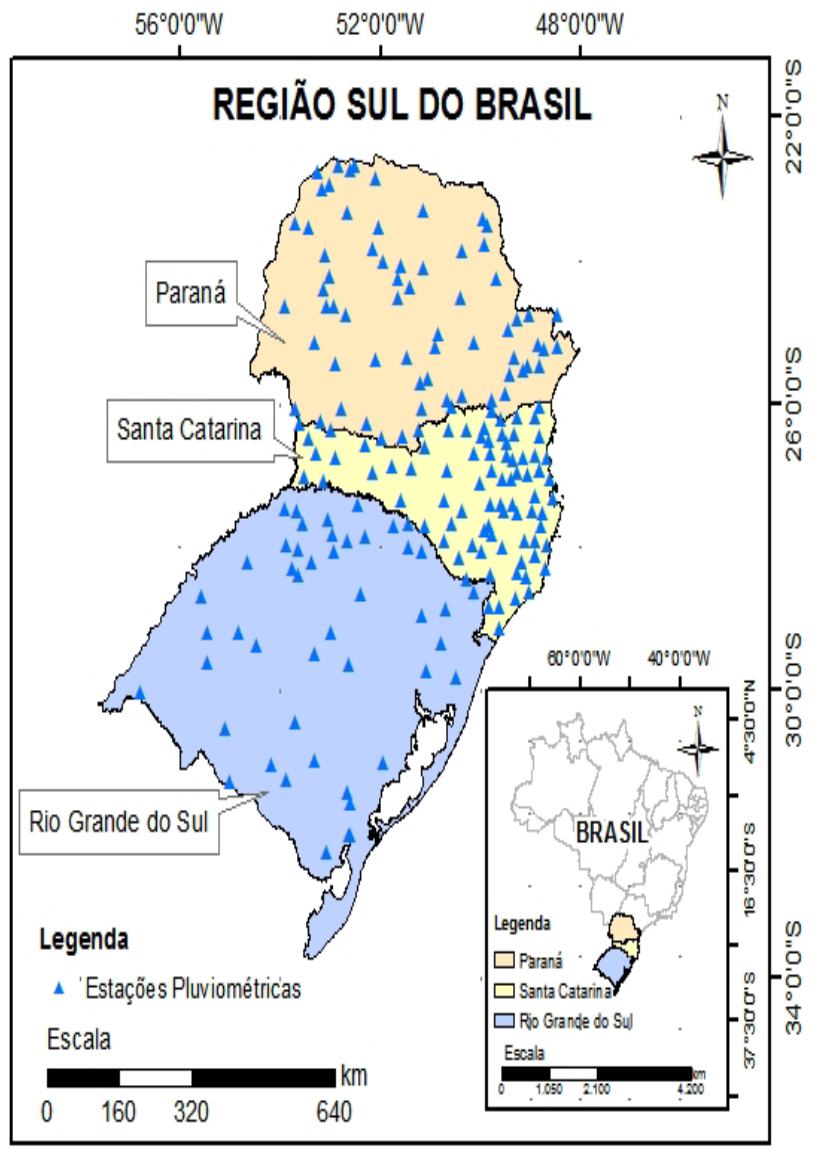

Figura 1 - Localização das estações pluviométricas selecionadas.
Com os valores de precipitação mensal foram constituídas as séries de precipitação por estação do ano e a precipitação total anual. Para o verão foi considerado o trimestre de Janeiro a Março e assim respectivamente para as demais estações.

O ICP foi proposto por Oliver em 1980 com a finalidade de determinar o grau de concentração ou a sazonalidade da precipitação. O ICP se baseia na distribuição mensal de chuvas, sendo recomendado para obtenção de informação sobre a variabilidade total da precipitação em um determinado do período temporal, sendo calculado em escalas anuais e sazonais. Quanto maior o seu valor, maior a concentração da precipitação. Esse índice é expresso pela Eq. 01:

$\mathrm{ICP}=100 * \frac{\sum_{\mathrm{i}=1}^{12} \mathrm{p}_{\mathrm{i}}^{2}}{\left(\sum_{\mathrm{i}=1}^{12} \mathrm{p}_{\mathrm{i}}^{2}\right)^{2}}$

onde, é a precipitação mensal no mês i.

O índice também pode ser calculado em escala sazonal (Eq. 02) para os meses correspondentes às estações do verão, outono, primavera e inverno (De Luis et al., 2011):

$$
\mathrm{ICP}_{\text {sazonal }}=\frac{\sum_{\mathrm{i=1}}^{3} \mathrm{p}_{\mathrm{i}}^{2}}{\left(\sum_{\mathrm{i}=1}^{3} \mathrm{p}_{\mathrm{i}}\right)^{2}} * 25
$$

Para uma escala suprasazonal, no período úmido, que compreende os meses de outubro a março e seco, de abril a setembro (De Luis et al., 2011) utiliza-se a seguinte fórmula (Eq. 03):

$$
\mathrm{ICP}_{\text {suprasazonal }}=\frac{\sum_{\mathrm{i}=1}^{6} \mathrm{p}_{\mathrm{i}}^{2}}{\left(\sum_{\mathrm{i}=1}^{6} \mathrm{p}_{\mathrm{i}}\right)^{2}} * 50
$$

Conforme equações do ICP sazonal e suprasazonal, o menor valor do ICP é de 8,3, indicando perfeita uniformidade na distribuição da precipitação. Assim como, valores próximos de 16,7 indicam que a precipitação total foi concentrada em meio do período e valores de ICP acima de 25 , que a precipitação total ocorreu em $1 / 3$ do período, o que significa que o total anual da precipitação ocorre em quatro meses. 
Para avaliar a hipótese de tendências nas dez séries estudadas de cada estação pluviométrica foi utilizado o teste não paramétrico de Mann-Kendall (Mann, 1945; Kendal, 1975) que é um método não paramétrico que vem sendo muito utilizado para analisar tendências em séries temporais (Penereiro \& Ferreira, 2011). Este teste é considerado um teste robusto, e por ser não paramétrico, não exige a normalidade dos dados (Wang, 2008), apresentando ainda a vantagem de ser pouco influenciado por mudanças abruptas ou séries não homogéneas (Back, 2001; Yue et al., 2002; Santos \& Portela, 2007; Zhang et al., 2009). Este teste é fortemente recomendado pela Organização Meteorológica Mundial (Yue et al., 2002; Huang et al., 2014) para detectar tendências em séries históricas de dados hidrológicos, climáticos bem como dos índices relacionados.

Para a aplicação desse método consideram-se duas hipóteses a serem testadas:

H0: Os dados constituem uma amostra aleatória de $\mathrm{N}$ valores independentes e identicamente distribuídos (ou seja, o conjunto de dados não apresenta tendência);

H1: A distribuição das variáveis $X i$ e $X j$ não é idêntica para pelo menos alguns $i, j \leq N$, com $i \neq j$, onde i e j são as sequências dos dados e $\mathrm{N}$ é a dimensão da série.

A estatística do método de Mann-Kendall (S) é dada pela Eq. 04:

$$
S=\sum_{j=1}^{N-1} \sum_{i=j+1}^{N} \operatorname{sgn}(X i-X j)
$$

em que:

$\operatorname{sgn}(\theta)=\left\{\begin{array}{c}1 \text { se } \theta>0 \\ 0 \text { se } \theta=0 \\ -1 \text { se } \theta<0\end{array}\right\}$

A média e variância de $S$, para variável aleatória distribuída sem tendências, são dadas pelas Equações 02 e 03:

$\mathrm{E}(\mathrm{S})=0$
$\operatorname{Var}(\mathrm{S})=\frac{\mathrm{N}(\mathrm{N}-1)(2 \mathrm{~N}+5)-\sum_{\mathrm{j}=1}^{\mathrm{p}} \mathrm{tj}(\mathrm{tj}-1)(2 \mathrm{tj}+5)}{18} \quad($ Eq. 06)

sendo $\mathrm{p}$ o número de empates e tj o número de pontos no grupo $\mathrm{j}$.

Fazendo-se o teste bilateral, em nível de significância $\alpha$, a hipótese $\mathrm{H} 0$ é rejeitada se o teste estatístico padronizado, $Z<Z \alpha / 2$ ou $Z>Z \alpha / 2$, onde a estatística normalizada $Z$ de Mann-Kendall segue aproximadamente a distribuição normal com média zero e variância um. $\mathrm{O}$ valor de $\mathrm{Z}$ é calculado por meio da Eq. 07:

$Z=\left\{\begin{array}{c}\frac{\mathrm{s}-1}{\sqrt{\operatorname{var}(\mathrm{S})}} \mathrm{seS}>0 \\ 0 \mathrm{seS}=0 \\ \frac{\mathrm{S}+1}{\sqrt{\operatorname{var}(\mathrm{S})}} \mathrm{seS}<0\end{array}\right\}$

Com o valor de $\mathrm{Z}$ determina-se a probabilidade de rejeitar a hipótese nula (valor $\mathrm{p}$ ), sendo esta verdadeira. Este valor foi avaliado tendo como nível de significância $5 \%(\alpha=0,05)$, para todos $i>j$.

\section{RESULTADOS E DISCUSSÃO}

Analisando as tendências das séries de dados obtidas através do teste Mann-Kendall (Figura 2 A) não foram observadas tendências significativas de precipitação para o período de 1976 a 2015, visto que para todo o sul do Brasil, apenas 2,5\% das estações apresentaram tendência significativa de diminuição no volume de precipitação e 9,6\% apresentaram tendência significativa de aumento.

O Quadro 1 apresenta as percentagens de tendências significativas positivas e negativas e tendências não significativas para cada estado, bem como para toda a região sul. No Paraná, das 69 estações analisadas, três $(4,3 \%)$ apresentaram tendência negativa com maior incidência no norte do estado e nove (13\%) apresentaram tendência positiva, sobretudo no sul do estado. Em Santa Catarina, das 75 (12\%) estações, nove apresentaram tendência positiva, principalmente no norte do estado, e nenhuma estação apresentou tendência negativa. No Rio Grande do Sul, das 53 estações analisadas, duas $(3,8 \%)$ apresentaram tendência 


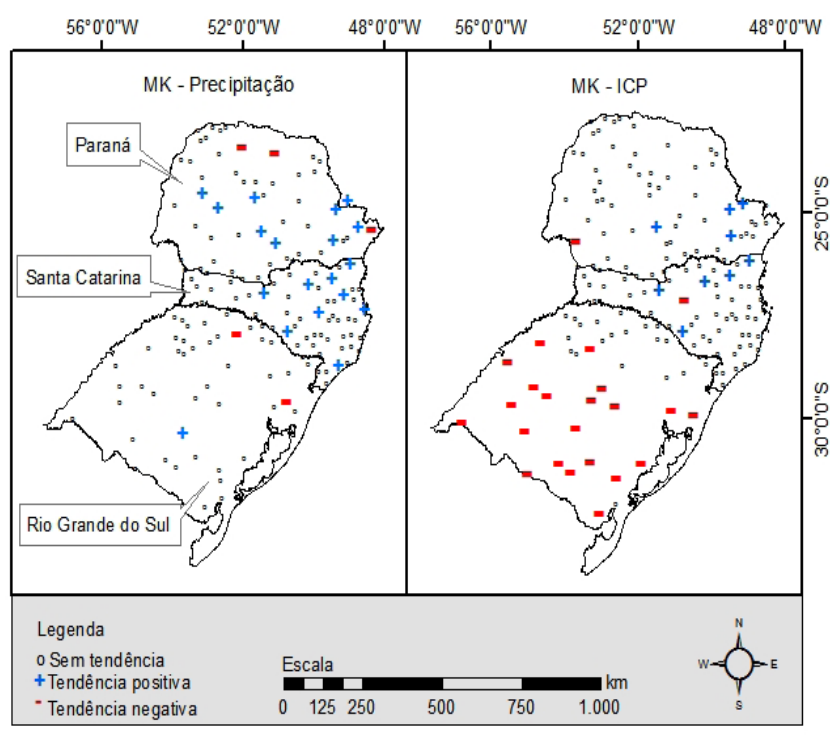

Figura 2 - Mapa de tendência de precipitação e Índice de Concentração de Precipitação anual, no período de 1976 a 2015, para a região sul do Brasil.

negativa e apenas uma (1,9\%) apresentou tendência positiva.

Constata-se que no período analisado a maioria das séries estudadas não apresentou tendências significativas na precipitação total anual. Alguns estudos realizados sobre tendências nas séries de precipitação da região Sul do Brasil identificaram tendências (Haylock et al., 2006; Pinheiro et al., 2013) com o teste de Mann-Kendall, Campos et al. (2006) encontraram um aumento dos totais anuais de precipitação no período entre 1911 e 2006 no estado de Santa Catarina. No Rio Grande do Sul, Sansigolo \& Kayano (2010) também apontaram tendências significativas de aumento, com uma variação de 93 mm em 100 anos, na média sazonal de verão.

Por outro lado, existem vários estudos recentes em que não foram identificadas tendências nas séries de precipitação da região sul do Brasil. Ricce et al. (2009) analisaram a tendência de precipitação no estado de Paraná e concluíram que não houve tendência significativa de precipitação, porém ressaltam que a distribuição da precipitação tem sido mais inconstante nos últimos anos. Machado (2017) analisando as séries de precipitação de 18 estações pluviométricas do estado do Paraná, não identificaram tendências significativas usando os testes de
Mann-Kendall, Pettitt e Sen. Silva et al. (2015) analisando séries de precipitação de 40 estações pluviométricas localizadas no rio Grande do Sul, com dados de 1961 a 2002, não identificaram tendências significativas nas séries de precipitação total anual a partir do teste de Mann-Kendall. Favaretto et al. (2016), analisando tendências de série de precipitação de Porto Alegre (RS) do período de 1961 a 2013, empregando vários testes não paramétricos incluindo o teste de Mann-Kendall, concluíram que as séries de precipitação trimestrais e anual não possuem tendências significativas. Milléo et al. (2015) analisando a série de precipitação de 1989 a 2013 de Curitiba (PR) concluíram que a série deve ser considerada estacionária.

Essa aparente divergência nos estudos pode ser devido ao diferentes períodos usado pelos autores. Pinheiro et al. (2013) utilizaram séries diferentes para cada estação, no entanto a maioria compreendida entre 1944 a 2006, Haylock et al. (2006) por sua vez utilizaram dados de 1960 a 2000. Back (2001), analisando a série de precipitação de Urussanga (SC) do período de 1924 a 1998 observou tendência significativa de aumento, da precipitação total anual. Por meio do teste de Pettitt o autor identificou que o ponto de mudança na série teria ocorrido no ano de 1971. Back et al. (2012), analisando a séries de dados de precipitação de 1955 a 2010 também de Urussanga encontraram tendências significativa de aumento da precipitação anual. Posteriormente Denski e Back (2015) analisando tendência nas séries climatológicas na evapotranspiração de referência de Urussanga, utilizando as séries do período de 1980 a 2013, não encontraram tendências significativas na série de precipitação total anual de Urussanga. Estes estudos mostram que, mesmo usando dados do mesmo local e o mesmo teste estatístico, os resultados podem ser diferentes em função do tamanho da série e do período de dados que compõem a série usada.

Referente ao teste de tendência aplicado ao Índice de Concentração de Precipitação (Figura 2 B), 4,6\% das estações estudadas apresentaram tendência positiva de precipitação e 11,7\% apresentaram tendência negativa. As tendências negativas se concentraram nas estações pluviométricas localizadas no sul do estado do Rio Grande do Sul. A redução no índice de concentração indica a ocorrência de melhor distribuição das chuvas durante o ano, 
Quadro 1 - Frequência (\%) de tendências anuais e sazonais

\begin{tabular}{|c|c|c|c|c|c|c|}
\hline \multirow[b]{2}{*}{ Estado } & \multicolumn{6}{|c|}{ Precipitação } \\
\hline & Tendência & Anual & Verão & Outono & Inverno & Primavera \\
\hline \multirow{3}{*}{ Paraná } & Positiva & 13 & 18,8 & 2,9 & 2,9 & 5,8 \\
\hline & Não significativa & 82,7 & 79,8 & 97,1 & 97,1 & 92,8 \\
\hline & Negativa & 4,3 & 1,4 & 0 & 0 & 1,4 \\
\hline \multirow{3}{*}{ Santa Catarina } & Positiva & 12 & 17,3 & 12 & 10,7 & 14,7 \\
\hline & Não significativa & 88 & 82,7 & 86,7 & 89,3 & 85,3 \\
\hline & Negativa & 0 & 0 & 1,3 & 0 & 0 \\
\hline \multirow{3}{*}{$\begin{array}{l}\text { Rio Grande } \\
\text { do Sul }\end{array}$} & Positiva & 1,9 & 1,9 & 1,9 & 1,9 & 1,9 \\
\hline & Não significativa & 94,3 & 98,1 & 98,1 & 90,6 & 98,1 \\
\hline & Negativa & 3,8 & 0 & 0 & 7,5 & 0 \\
\hline \multirow{3}{*}{ Região Sul } & Positiva & 9,6 & 13,7 & 6,1 & 5,6 & 8,1 \\
\hline & Não significativa & 87,9 & 85,8 & 93,4 & 92,4 & 91,4 \\
\hline & Negativa & 2,5 & 0,5 & 0,5 & 2 & 0,5 \\
\hline & \multicolumn{6}{|c|}{ ICP } \\
\hline Estado & Tendência & Anual & Verão & Outono & Inverno & Primavera \\
\hline \multirow{3}{*}{ Paraná } & Positiva & 5,8 & 5,8 & 2,9 & 10,1 & 11,6 \\
\hline & Não significativa & 92,8 & 92,8 & 92,8 & 88,5 & 87 \\
\hline & Negativa & 1,4 & 1,4 & 4,3 & 1,4 & 1,4 \\
\hline \multirow{3}{*}{ Santa Catarina } & Positiva & 6,7 & 10,7 & 10,7 & 9,3 & 12 \\
\hline & Não significativa & 92 & 89,3 & 86,6 & 90,7 & 88 \\
\hline & Negativa & 1,3 & 0 & 2,7 & 0 & 0 \\
\hline \multirow{3}{*}{$\begin{array}{l}\text { Rio Grande } \\
\text { do Sul }\end{array}$} & Positiva & 0 & 0 & 0 & 3,8 & 1,9 \\
\hline & Não significativa & 60,4 & 86,8 & 90,6 & 88,7 & 98,1 \\
\hline & Negativa & 39,6 & 13,2 & 9,4 & 7,5 & 0 \\
\hline \multirow{3}{*}{ Região Sul } & Positiva & 4,6 & 6,1 & 5 & 7,6 & 9,1 \\
\hline & Não significativa & 83,7 & 89,8 & 90 & 89,7 & 90,4 \\
\hline & Negativa & 11,7 & 4,1 & 5 & 2,5 & 0,5 \\
\hline
\end{tabular}

que traz como benefícios indiretos a redução nos processos erosivos e diminuição das frequências de estiagens. Este comportamento diferenciado no extremo da região Sul do Brasil deve ser melhor averiguado em futuros trabalhos, uma vez que não foram encontrados estudos anteriores relatando mudanças na concentração das chuvas nesta região. Winke et al. (2008), ao analisarem dados de precipitação e de temperatura do município de Pelotas, no período de 1931 a 2007, observaram tendência crescente na temperatura mínima anual, porém nenhuma tendência significativa na precipitação. Diaz et al. (1998) estudaram a precipitação no Uruguai e no Estado do Rio Grande do Sul, utilizando dados de 40 estações pluviométricas no período de 1917-1980 e concluíram que houve maior variabilidade de precipitação do interior para o litoral (oeste-leste), com maior valor positivo em relação à média anual, no oeste, e no leste ao longo da costa atlântica. Já na direção norte-sul, os maiores desvios positivos em relação à média anual ocorreram no sul, durante o fim do verão e outono, e durante o início da primavera e início do verão, no norte. Em geral, o estudo mostrou que a variabilidade de precipitação no Uruguai e no Rio Grande do Sul está associada às anomalias da temperatura da superfície do mar, em ambos os oceanos (Pacífico e Atlântico).

Silva et al. (2010) estudaram séries temporais de precipitação para o Brasil num período de 1961 a 2008, e concluíram que há padrões diferenciados e bastante regionalizados nas tendências de aumento e diminuição das precipitações. Numa avaliação anual, os autores verificaram que no leste da região sul do Brasil há tendências positivas mais significativas, desde o centro do Rio Grande do Sul até o Paraná. A redução dos valores de ICP no Rio Grande do Sul pode ser interpretada como sendo de efeito positivo, visto que a média anual desse índice foi igual a 11,06, o que, segundo a classificação de Michiels et al. (1992), indica uma distribuição 
de precipitação moderadamente sazonal. Quanto menor o valor do ICP melhor é a sua distribuição sazonal, ou seja, a precipitação é mais uniforme ao longo do ano, o que implica em menores riscos de estiagens e de erosão do solo (Gil, 2012; Viola et al., 2014).

Avaliando-se as análises sazonais de tendência de precipitação, observa-se comportamento semelhante à série anual, com predomínio de séries estacionárias (tendências não significativas). $O$ verão foi a estação que apresentou maior frequência de tendências positivas (Figura 3), correspondendo a $13,2 \%$ das estações estudadas.

No estado do Paraná foi observado maior número de tendência de aumento de precipitação (18\% das estações do estado) no verão, assim como no estado de Santa Catarina (17\% das estações do estado).

No estado do Rio Grande do Sul apenas uma estação apresentou tendência de aumento de precipitação

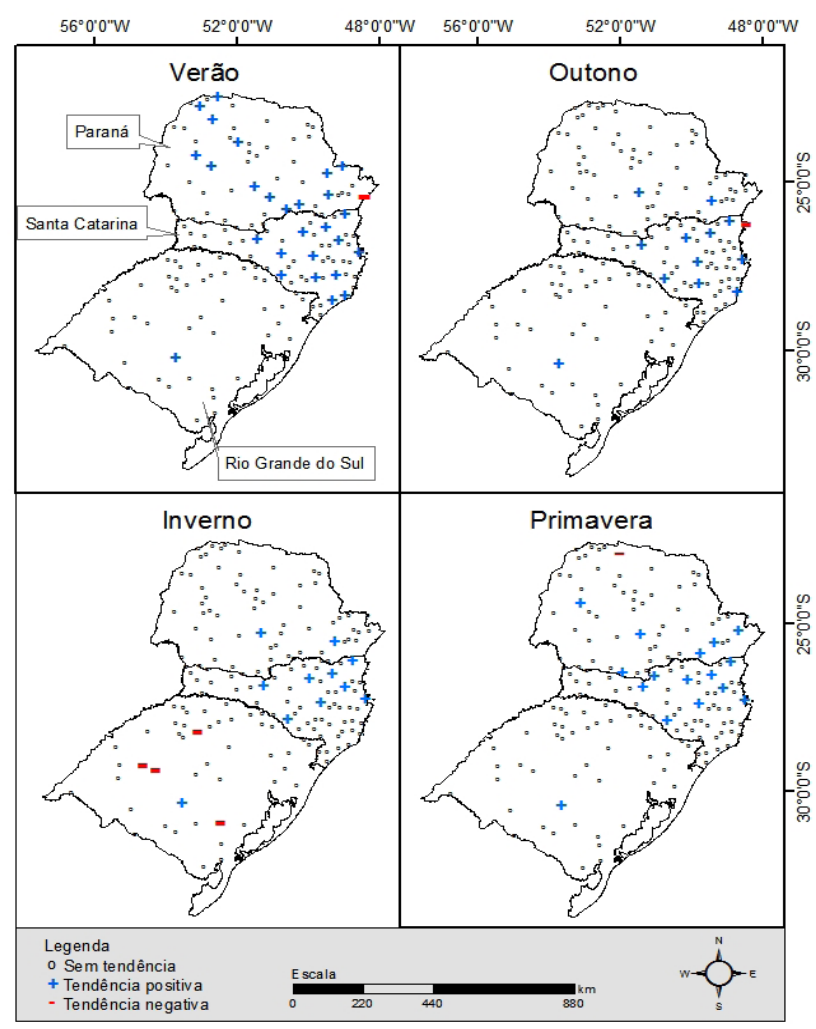

Figura 3 - Mapa sazonal de tendência de precipitação, no período de 1976 a 2015, para a região sul do Brasil. em todas as estações. O que não representa para o estado aumento no volume de precipitação para a série estudada.

O verão, outono e inverno apresentaram apenas uma estação pluviométrica com tendência negativa. Na primavera o estado do Rio Grande do Sul apresentou 7,5\% de estações com tendência na redução do volume de precipitação. Em estudo sobre a tendência da precipitação sazonal sobre o sul do Brasil, Sabatini (2008) verificou que apenas no verão houve tendências negativas, para as outras estações as tendências foram positivas. Contudo, os resultados de Sansigolo e Kayano (2010) mostram tendência de aumento da precipitação para o Rio Grande do Sul durante o período de 1913 a 2006, principalmente no verão.

Ao estudar a variabilidade interanual das precipitações no estado do Rio Grande do Sul, Nery et al. (1997) observaram que todas as flutuações interanuais mais significativas da precipitação estiveram associadas com as fases extremas da Oscilação Sul, e que a seca nesta região nem sempre está associada com o fenômeno La Niña. Fontana e Berlato (1997) também constataram que há influência do ENOS na distribuição da precipitação pluvial, a qual é mais intensa na porção oeste do estado do Rio Grande do Sul nos meses de outubro e novembro. No ano de 1988/89 o fenômeno La Niña foi responsável pela forte estiagem que assolou o estado na safra de verão, em 1990/91.

Em termos espaciais os resultados não mostraram a ocorrências de zonas com tendências significativas, mostrando que as tendências significativas observadas foram em pequeno número de estações e que ocorrem aleatoriamente. Este fato também reforça a constatação de não haver tendência significativas nas séries de precipitação na região Sul do Brasil no período analisado.

Os resultados do teste de Mann-Kendall aplicado ao Índice de Concentração de Precipitação (Figura 4) demonstram que no verão as tendências de aumento na concentração de precipitação $(6,1 \%)$ acontecem nos estados do Paraná e Santa Catarina. Já as tendências negativas $(4,1 \%)$, que representam mais uniformidade na concentração das chuvas, concentraram-se no estado do Rio Grande do Sul. 


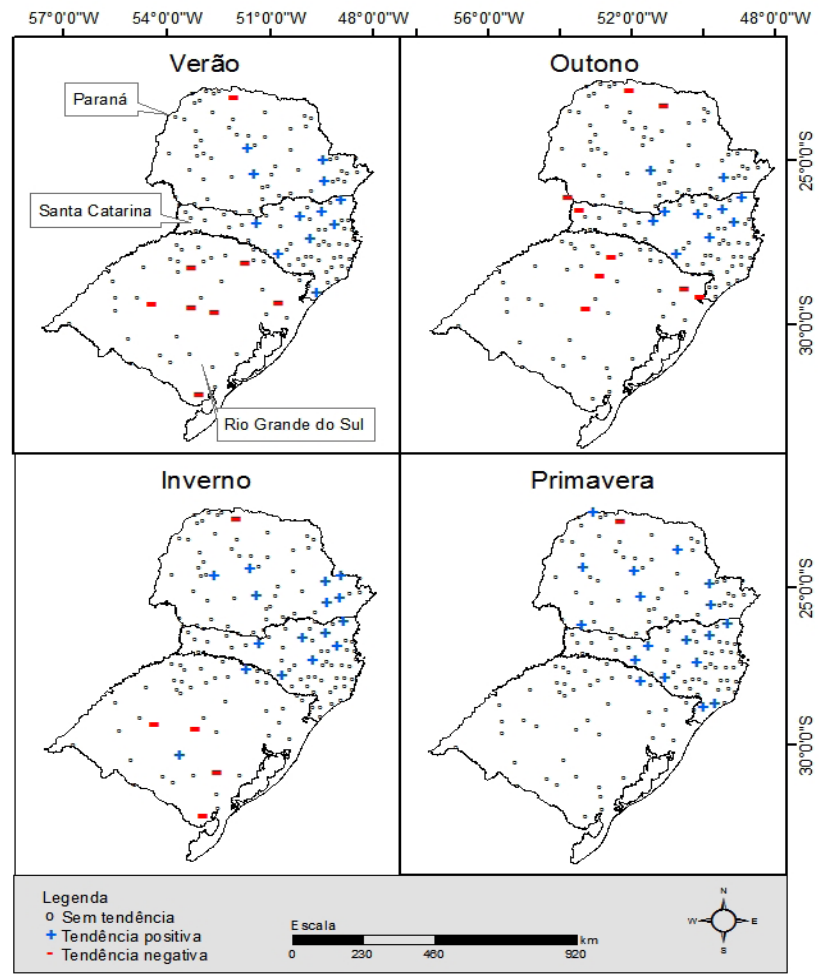

Figura 4 - Mapa sazonal de tendência do Índice de Concentração de Precipitação, no período de 1976 a 2015, para a região sul do Brasil.

As variações sazonais e espaciais tanto nas tendências pluviométricas quanto do ICP devem-se à atuação de mecanismos responsáveis pela formação das chuvas na região Sul do Brasil, visto que essa região, segundo Grimm (2009), apresenta grandes contrastes no regime de precipitação, com transição bem clara onde ao norte domina o típico regime de monção, com a estação chuvosa iniciando-se na primavera e terminando no início do outono, resultando em grandes diferenças entre o verão e outono, enquanto no sul há distribuição aproximadamente uniforme de chuvas ao longo do ano, com chuvas mais fortes no inverno. $\mathrm{Na}$ estação do verão ocorre aquecimento da superfície e condução de umidade para o interior do continente, o que tende a instabilizar a atmosfera, aumentando os processos convectivos, resultando em chuvas intensas mais ao norte da região Sul. Tanto no verão como nas estações de transição os Complexos Convectivos de Mesoescala (CCM) são frequentes e responsáveis por grande parte da precipitação total. No inverno ocorre maior penetração de frentes no Rio Grande do Sul, provocando maior quantidade de chuvas no Sudeste da região Sul. Na costa leste da Região Sul, especialmente no Paraná, há significativa contribuição do efeito orográfico para a precipitação. No sudeste do Rio Grande do Sul o máximo de precipitação ocorre no inverno, quando as chuvas são resultantes de penetrações frontais associadas a ciclones extratropicais migratórios. No nordeste da Região Sul abrangendo o estado do Paraná e de Santa Catarina predomina chuvas de verão com o máximo no trimestre dezembro a fevereiro ou janeiro a março. No oeste a maior precipitação ocorre na primavera, mais ao norte e no outono mais ao sul. Apenas no Rio Grande do Sul o trimestre mais chuvoso é de julho a setembro.

O litoral de Santa Catarina apresenta ocorrência de eventos extremos de precipitação (Haas, 2002; Lima et al., 2009; Silva Dias, 2009). Entre os mecanismos formadores da precipitação destacam-se o deslocamento das frentes frias e ciclones. Estes sistemas ciclónicos também estão entre os principais sistemas atmosféricos que contribuem para a precipitação nos estados do Sul do Brasil. Em estudos realizados sobre a climatologia da precipitação em Santa Catarina, a estação de verão aparece como a mais chuvosa nas áreas litorâneas e do Vale do Itajaí (Nimer, 1979; Grimm et al., 1998).

\section{CONCLUSÕES}

No período de 1975 a 2015, das 197 estações estudadas, $87,9 \%$ não apresentaram tendências significativas de aumento ou redução no volume de precipitação anual. Enquanto que 9,6 \% das estações apresentaram tendências positivas e 2,5\% com tendências negativas, indicado que a séries de precipitação da região Sul do Brasil são estacionárias.

Não foi possível caracterizar a influência das mudanças climáticas nas séries climáticas estudadas.

Não foi observada variação espacial significativa nas tendências das séries de precipitação anual.

Nas séries sazonais foram observadas maiores ocorrência de tendências positivas nas séries do verão, principalmente no estado do Paraná e Santa Catarina 
Nas séries do ICP foi observado que no extremo sul do Rio Grande do Sul ocorrem tendências de diminuição do Índice de Concentração da precipitação, indicando a ocorrência de chuvas com melhor distribuição sazonal.

\section{AGRADECIMENTOS}

Os autores agradecem à Fundação de Apoio à Pesquisa Científica e Tecnológica do Estado de Santa Catarina (FAPESC) e ao Conselho Nacional de Desenvolvimento Científico e Tecnológico (CNPq).

\section{REFERÊNCIAS BIBLIOGRÁFICAS}

ANA (2009) - Inventário das estações pluviométricas. Agência Nacional de Águas. 2 ed. - Brasília: ANA; SGH. Back, A.J. (2001) - Aplicação de análise estatística para identificação de tendências climáticas. Pesquisa Agropecuária Brasileira, vol. 36, n. 5, p. 717-726. http://dx.doi.org/10.1590/S0100-204X2001000500001

Campos, C.; Alves, R. \& Braga, H.J. (2006) - Mudanças climáticas atuais e seus impactos no Estado de Santa Catarina. Agropecuária Catarinense, vol. 19, p. 31-35.

Cera, J.C.; Ferraz, S.E.T.; Bender, F.D. (2009) - Influência da Oscilação Decadal do Pacífico e as Mudanças no Regime de Chuva do Rio Grande do Sul. Ciência e Natura, vol. 1, p. 317-320.

Cera, J.C. \& Ferraz, S.E.T. (2015) - Variações climáticas na precipitação no sul do Brasil no clima presente e futuro. Revista Brasileira de Meteorologia, vol. 30, n. 1, p. 81-88. http://dx.doi.org/10.1590/0102-778620130588

De Luis, M.; González-Hidalgo, J.C.; Brunetti, M. \& Longares, L.A. (2011) - Precipitation concentration changes in Spain 1946-2005. Natural Hazards and Earth System Science, vol. 11, n. 5, p. 1259-1265. https://doi. org/10.5194/nhess-11-1259-2011

Denski, A.O.N. \& Back, A.J. (2015) - Tendência climatológica nos elementos meteorológicos e na evapotranspiração de referência de Urussanga-SC. Revista Brasileira de Climatologia, vol. 17, p. 262-274. http://dx.doi. org/10.5380/abclima.v17i0.38400

Diaz, A.F.; Studzinski, C.D. \& Mechoso, C.R. (1998) - Relationships between Precipitation Anomalies in Uruguay and Southern Brazil and Sea Surface Temperature in the Pacific and Atlantic Oceans. Journal of Climate, vol. 11, p. 251-271. https://doi.org/10.1175/1520-0442(1998)011<0251:RBPAIU>2.0.CO;2

Ely, D.F.; Almeida, I.R. \& Sant'Anna Neto, J.L. (2003) - Implicações políticas e econômicas, variabilidade climática e o rendimento da cultura do milho no estado do Paraná. Geografia, v. 12, n. 1, p. 495-508.

Favaretto, J.R.; Borba, W.F.; Allasia, D.G.; Silva, J. L.S. \& Lasta, L. (2016). - Análise de tendências em uma série de precipitação. Estudo de caso: Porto Alegre -RS. In: V Congresso Internacional de Tecnologias para o Meio Ambiente, Bento Gonçalves, p.1-8.

Fontana, D.C. \& Berlato, M.A. (1997) - Influência do El Niño Oscilação Sul sobre a precipitação do Estado do Rio Grande do Sul. Revista Brasileira de Agrometeorologia, vol. 5, n. 1, p. 127-132.

Gil, H.A.P. (2012) - El índice de erosión potencial en la vertiente norte del Waraira Repano, estado Vargas, Venezuela. Cuadernos de Geografía: Revista Colombiana de Geografía, vol. 21, n. 2, p. 85-97.

Grimm, A.M. (2009) - Clima da região Sul do Brasil, In: Cavalcanti, I.F.A. (Ed.) - Tempo e Clima no Brasil. São Paulo, Oficina Textos. p. 259-275.

Grimm, A.M.; Ferraz, S.E.T. \& Gomes, J. (1998) - Precipitation Anomalies in Southern Brazil Associated with El Niño and La Niña Events. Journal of Climate, vol. 11, n. 11, p. 2863-2880. https://doi.org/10.1175/ 1520-0442(1998)011<2863:PAISBA>2.0.CO;2

Haas, R. (2002) - Simulações da chuva orográfica associada a um ciclone extratropical no Litoral sul do Brasil. Tese de Doutorado. São Paulo, Universidade de São Paulo. 169 p.

Haylock, M.R.; Peterson, T.C.; Alves, L.M.; Ambrizzi, T.; Anunciação, Y.M.T.; Baez, J.; Barros, V.R.; Berlato, M.A.M.; Bidegain M.A.M.; Coronel, G.; Corradi, V.; Garcia, V.J.; Grimm, A.M.; Karoly, D.; Marengo, J.A.; Marino, M.B.; Moncunill, D.F.; Nechet, D.; Quintana, J.; Rebello, E.; Rusticucci, M.; Santos, J.L.; Trebejo, I. \& Vincent, L.A. (2006) - Trends in total and extreme South American rainfall 1960-2000 and links with sea surface temperature. Journal of Climate, vol. 19, p. 1490-1512. https://doi.org/10.1175/JCLI3695.1 
Huang, J.; Sun, S.; Xue, Y. \& Zhang, J. (2014) - Spatial and temporal variability of precipitation indices during 1961-2010 in Hunan Province, central south China. Theoretical and Applied Climatology, vol. 118, n. 3, p. 581-595. https://doi.org/10.1007/s00704-013-1087-6

Kendall, M.G. (1975) - Rank Correlation Methods, 4th ed., Charles Griffin: London.

Lima, M.; Rodrigues, M.L.G.; Sacco, F.; Araújo, G. \& Alves, M. (2009) - Análise da configuração atmosférica associada a eventos extremos de chuva no litoral do Estado de Santa Catarina, Sul do Brasil. In: III Simpósio Internacional de Climatologia. Canela, RS, Sociedade Brasileira de Meteorologia.

Machado, M.A.M. (2017) - Estimativa da erosividade de chuvas para o Estado do Paraná sob cenários de mudanças climáticas: Distribuição anual, sazonalidade e uso de modelos climáticos. Curitiba, Tese de Doutorado. 159 p.

Mann, H.B. (1945) - Non-parametric test against trend. Econometrica, vol. 13, n. 3, p. 245-259.

Marengo, J.A.; Ambrizzi, T; Soares, W.R. (2009) - Jato de Baixos Níveis ao longo dos Andes. In: Cavalcanti, I.F.A. et al. (Eds.) - Tempo e Clima no Brasil. São Paulo, Oficina de Textos, p. 169-180.

Michiels, P.; Gabriels, D. \& Hartmann, R. (1992) - Using the seasonal and temporal precipitation concentration index for characterizing monthly rainfall distribution in Spain. Catena, vol. 19, n. 1, p. 43-58. https:// doi.org/10.1016/0341-8162(92)90016-5

Milléo, C.; Ploszai, R. \& Mine, M.R.M. (2015) - Análise de tendências na série histórica de precipitações em Curitiba. In: XXI Simpósio Brasileiro de Recursos Hídricos, Brasília, p.1-8.

Monteiro, M.A. (2001) - Caracterização climática do estado de Santa Catarina: uma abordagem dos principais sistemas atmosféricos que atuam durante o ano. Geosul, vol.16, n. 31, p. 69-78. https://doi.org/10.5007/\%25x

Nery, J.T.; Vargas, M.W. \& Martins, M.L.O.F. (1997) - Variabilidade Interanual da Precipitação do Paraná. Revista Brasileira de Agrometeorologia, vol. 5, n. 1, p. 115-125.

Nimer, E. (1979) - Climatologia do Brasil. Rio de Janeiro: IBGE. 422 p.

Oliver, J.E. (1980) - Monthly precipitation distribution: A comparative index. The Professional Geographer, vol. 32, n. 3, p. 300-309. https://doi.org/10.1111/j.0033-0124.1980.00300.x

Pellegrino, G.Q.; Assad, E.D. \& Marin, F.R. (2007) - Mudanças Climáticas Globais e a Agricultura no Brasil. Revista Multiciência, n. 8.

Penereiro, J.C. \& Ferreira, D.H.L. (2011) - Estatística apoiada pela tecnologia: uma proposta para identificar tendências climáticas. Acta Scientiae, v. 13, n. 1, p. 87-105.

Pinheiro, A.; Graciano, R.L.G. \& Severo, D.L. (2013) - Tendência das séries temporais de precipitação da região sul do Brasil. Revista Brasileira de Meteorologia, vol. 28, n. 3, p. 281-290. http://dx.doi.org/10.1590/S010277862013000300005

Queiroz, M.A. (2013) - Avaliação de tendências em séries de precipitação diária máxima anual na faixa central do Estado de Minas Gerais. Dissertação (Mestrado em Saneamento, Meio Ambiente e Recursos Hídricos) - Escola de Engenharia da UFMG. Belo Horizonte. 202 p.

Ricce, W.S.; Caramori, P.H.; Morais, H.; Silva, D.A.B. \& Ataíde, L.T. (2009) - Análise de tendências na temperatura e precipitação em londrina, estado do Paraná. In: XV Congresso Brasileiro de Agrometeorologia. Belo Horizonte, MG.

Sabatini, A.D. (2008) - Índices de extremos climáticos de temperatura e chuva na América do Sul: clima presente e validação do modelo RegCM3. Dissertação (Mestrado em Meteorologia). São Paulo, Universidade de São Paulo.120 p.

Sansigolo, C.A. \& Kayano, M. T. (2010) - Trends of seasonal maximum and minimum temperatures and precipitation in Southern Brazil for the 1913-2006 period. Theoretical and Applied Climatology, vol. 101, n. 1-2, p. 209-216. https://doi.org/10.1007/s00704-010-0270-2

Santos, J.F. \& Portela, M.M. (2007) - Tendências em séries de precipitação mensal em Portugal continental. Aplicação do teste de Mann-Kendall. Simpósio de Hidráulica e Recursos Hídricos dos Países de Língua Oficial Portuguesa. APRH. São Paulo.

Silva Dias, M.A.F. (2009) - As chuvas de novembro de 2008 em Santa Catarina: Um estudo de caso visando à melhoria do monitoramento e da previsão de eventos extremos. Relatório Técnico INPE-16631-RPQ/255, 67 p.

Silva, F.D. dos S.; Ferreira, D.B.; Sarmanho, G.F.; Santos, L.S.F.C. dos; Fortes, L.T.G. \& Parente, E.G.P. (2010) - Tendência de alterações climáticas da precipitação observadas no Brasil de 1961 a 2008 utilizando dados gradeados. In: XII Congresso Brasileiro de Meteorologia, Belém. 
Silva, G.M.; Teixeira-Gandra, C.F.A.; Damé, R.C.F.; Klumb, G.B. \& Veber, P.M. (2015) - Tendências de séries de precipitação total mensal para localidades do Rio Grande do Sul. Revista Brasileira de Engenharia e Sustentabilidade, vol. 1, n. 2, p. 13-22.

Tucci, C.E.M. (2004) - Hidrologia: ciência e aplicação. 4 ed. Porto Alegre: Ed. Universidade/UFRGS, 943 p.

UFSC (2016) - Relatório dos danos matérias e prejuizos decorrentes de desastres naturais em Santa Catarina. 1995 - 2014. Universidade Federal de Santa Catarina. Centro Universitário de Estudos e Pesquisas sobre Desastres Florianópolis: CEPED UFSC. 72p.

Viola, M.R.; Avanzi, J.C.; Mello, C.R. de; Lima, S.O. \& Alves, M.V.G. (2014) - Distribuição e potencial erosivo da das chuvas no Estado de Tocantins. Pesquisa Agropecuária Brasileira, vol. 49, n. 2, p. 125-135.

Wang, M. (2008) - A remark on Andrews-Askey integral. Journal of Mathematical Analysis and Applications, vol. 341, n. 2, p. 1487-1494. https://doi.org/10.1016/j.jmaa.2007.11.011

Winke, L.O.L.; Damé, R.C.F. Teixeira, C.F.A.; Machado, A.A. \& Rosskoff, J.L.C. (2008) - Caracterização climática e estudo de tendências nas séries temporais de temperatura e precipitação em Pelotas/RS. In: XVII Congresso de Iniciação Científica. X Encontro de PósGraduação. São Paulo, Brasil.

Yue, S.; Pilon, p. \& Cavadias, G. (2002) - Power of the Mann-Kendall and Spearman's rho tests for detecting monotonic trends in hydrological series. Journal of Hydrology, vol. 259, n. 1-4, p. 254-271. https://doi. org/10.1016/S0022-1694(01)00594-7

Zhang, W.; Yan, Y.; Zheng, J.; Li, L.; Dong, X. \& Cai, H. (2009) - Temporal and spatial variability of annual extreme water level in the Pearl River Delta region, China. Global and Planetary Change, vol. 69, n. 1-2, p.35-47. https://doi.org/10.1016/j.gloplacha.2009.07.003 